International Journal of Pure and Applied Mathematics

Volume 102 No. 4 2015, 747-755

ISSN: 1311-8080 (printed version); ISSN: 1314-3395 (on-line version)

url: http://www.ijpam.eu

doi: http://dx.doi.org/10.12732/ijpam.v102i4.12

\title{
THE ORBIT GRAPH OF FINITE NON-ABELIAN GROUPS
}

\author{
S.M.S. Omer ${ }^{1}$, N.H. Sarmin 2 , A. Erfanian ${ }^{3}$ \\ ${ }^{1}$ Department of Mathematics \\ Faculty of Science \\ University of Benghazi \\ Benghazi, Libya \\ ${ }^{2}$ Department of Mathematical Sciences \\ Faculty of Science \\ Universiti Teknologi Malaysia \\ Johor Bahru, MALAYSIA \\ ${ }^{3}$ Department of Mathematics and Center of Excellence \\ in Analysis on Algebraic Structures \\ Ferdowsi University of Mashhad \\ Mashhad, IRAN
}

\begin{abstract}
Let $G$ be a finite non-abelian group and let $\Omega$ be a set of elements of $G$. Let $A$ be the set of commuting elements in $\Omega$, i.e $A=\{v \in \Omega: v g=$ $g v, g \in G\}$. In this paper, we extend the work on conjugate graph by defining a new graph called the orbit graph, denoted as $\Gamma_{G}^{\Omega}$. The vertices of $\Gamma_{G}^{\Omega}$ are non central elements in $\Omega$ but not in $A$ in which two vertices of $\Gamma_{G}^{\Omega}$ are adjacent if they are conjugate. Some graph properties are provided. Besides, the orbit graph of dihedral groups and quaternion groups is determined.
\end{abstract}

AMS Subject Classification: 20P05, 20B40, 97K30

Key Words: commutativity degree, graph theory, group action, conjugate graph

Received: March 29, 2015

(C) 2015 Academic Publications, Ltd. url: www.acadpubl.eu

${ }^{\S}$ Correspondence author 


\section{Introduction}

Graph theory is the study of vertices and edges. More precisely, it involves the ways in which sets of points can be connected by edges. The concept in graph theory is widely used among many fields and one of these uses are in group theory. In this section, some basic definitions that are needed in this paper are stated, starting with some definitions related to graph theory that can be found in one of the references ([1] and [2]). A graph $\Gamma$ is a mathematical structure consisting of two sets namely vertices and edges which are denoted by $V(\Gamma)$ and $E(\Gamma)$, respectively.

A graph is called directed if its edges are identified with ordered pair of vertices. Otherwise, $\Gamma$ is called indirected. Two vertices are adjacent if they are linked by an edge. A connected graph is a graph in which there is a partition of vertex $V$ into non empty subsets, $V_{1}, V_{2}, \ldots, V_{n}$ such that two vertices $\omega_{1}$ and $\omega_{2}$ are connected if and only if they belong to the same set $V_{i}$. Subgraphs $\Gamma\left(V_{1}\right), \Gamma\left(V_{2}\right), \ldots, \Gamma\left(V_{n}\right)$ are all components of $\Gamma$. The graph $\Gamma$ is connected, if it has precisely one component. Thus, a subgraph of a graph $\Gamma$ is a graph whose vertices and edges are subset of the vertices and edges of $\Gamma$. Hence we denote $\Gamma_{\text {sub }}$ a subgraph of $\Gamma$. However, a complete graph is a graph where each ordered pair of distinct $n$ vertices are adjacent, and it is denoted by $K_{n}$.

Next, we state some graph properties that are needed in this paper: A nonempty set $S$ of $\mathrm{V}(\Gamma)$ is called an independent set of $\Gamma$ if there is no adjacent between two elements of $S$ in $\Gamma$, whilst the independence number is the number of vertices in maximum independent set and it is denoted by $\alpha(\Gamma)$. However, the maximum number $c$ for which $\Gamma$ is $c$-vertex colorable is known as chromatic number and is denoted by $\chi(\Gamma)$. The diameter is the maximum distance between any two vertices of $\Gamma$ and $d(\Gamma)$ is used as a notation.

Furthermore, a clique is a complete subgraph in $\Gamma$, while the clique number is the size of the largest clique in $\Gamma$ and is denoted by $\omega(\Gamma)$. The dominating set $X \subseteq V(\Gamma)$ is a set where for each $v$ outside $X$, there exists $x$ in $X$ such that $v$ adjacent to $x$. The minimum size of $X$ is called the dominating number denoted by $\gamma(\Gamma)([1]$ and [2]).

Since this work is a connection between group theory and graph theory, hence we provide some works related to group theory, more precisely to the commutativity degree and one of its extensions, namely the probability that a group element fixes a set. The commutativity degree is the probability that two random elements of a group commute [3]. This concept has then been generalized and extended by several authors. One of the extensions is the probability that a group element fixes a set introduced by Omer et al. [4], in 
which the probability is ratio of number of orbits under group action to the order of the set. The following theorem is one of their work.

Theorem 1. [4] Let $G$ be a finite group. Let $S$ be a set of elements of $G$ of size two in the form of $(a, b)$ where $a$ and $b$ commute and $\operatorname{lcm}(|a|,|b|)=2$. Let $\Omega$ be the set of all subsets of commuting elements of $G$ of size two and $G$ acts on $\Omega$. Then the probability that an element of a group fixes a set is given by:

$$
P_{G}(\Omega)=\frac{K(\Omega)}{|\Omega|}
$$

where $K(\Omega)$ is the number of orbits of $\Omega$ in $G$.

The probability is found for some finite non-abelian groups include metacyclic 2-groups, dihedral groups, symmetric groups and alternating groups.

This paper is divided into three sections. The first section focuses on some background topics on graph theory and algebra, while the second section provides some earlier and recent publications that are related to the probability that a group element fixes a set and some graphs. In the third section, we present our main results on which include the orbit graph.

\section{Preliminaries}

In this section, some works that are related to the probability that an element of a group fixes a set and graph theory are stated. We commence with brief information about the probability of a group element fixes a set, followed by some related work on graph theory.

In 2013, the probability that a group element fixes a set was introduced by Omer et al [4]. The work in [4] has then been extended by finding the probability for some finite non-abelian groups including dihedral group, quaternion groups, symmetric groups and alternating groups [4], [5] and [6]. The following are some results that are needed in this paper.

Theorem 2. [4] Let $G$ be a dihedral group of order $2 n$, where $n \in \mathbb{N}$. Let $S$ be a set of elements of $G$ of size two in the form of $(a, b)$ where $a$ and $b$ commute and $\operatorname{lcm}(|a|,|b|)=2$. Let $\Omega$ be the set of all subsets of commuting elements of size two. If $G$ acts on $\Omega$ by conjugation, then

$$
P_{G}(\Omega)=\left\{\begin{array}{l}
\frac{6}{m+1}, \text { if } n \text { is even, } \frac{n}{2} \text { is odd and } m=\frac{5 n}{2}, \\
\frac{7}{m+1}, \text { if } n \text { is even, } \frac{n}{2} \text { is even and } m=\frac{5 n}{2}, \\
\frac{1}{n}, \quad \text { if } n \text { is odd } .
\end{array}\right.
$$


Theorem 3. [4] Let $G$ be the quaternion group $G \cong\left\langle a, b: a^{2^{n-1}}=1, b^{2}=\right.$ $\left.a^{2^{n-2}}, b a b^{-1}=a^{-1}\right\rangle$. Let $S$ be a set of elements of $G$ of size two in the form of $(a, b)$ where $a$ and $b$ commute and $\operatorname{lcm}(|a|,|b|)=2$. Let $\Omega$ be the set of all subsets of commuting elements of $G$ of size two and $G$ acts on $\Omega$ by conjugation. Then $P_{G}(\Omega)=1$.

Next, some relevant works on graph theory. In 1990, Bertram et al. [7] introduced a graph which is called a graph related to conjugacy classes. The vertices of this graph are non-central conjugacy classes, where two vertices are adjacent if the cardinalities are not coprime. Recently, Bianchi et al. [8] studied the regularity of the graph related to conjugacy classes and provided some results.

In 2013, Moradipour et al. [9] used the graph related to conjugacy classes to find some graph properties of some finite metacyclic 2-groups. However, Erfanian and Tolue [10] introduced a new graph which is called a conjugate graph. The vertices of this graph are non-central elements of a finite nonabelian group. Two vertices of this graph are adjacent if they are conjugate.

\section{Main Results}

In this section, we introduce our results, starting with the definition of orbit graph.

Definition 4. Let $G$ be a finite group and $\Omega$ be a set of elements of $G$. Let $A$ be the set of commuting elements in $\Omega$, i.e $A=\{v \in \Omega: v g=g v, g \in G\}$. The orbit graph $\Gamma_{G}^{\Omega}$ consists of two sets, namely vertices and edges denoted by $V\left(\Gamma_{G}^{\Omega}\right)$ and $E\left(\Gamma_{G}^{\Omega}\right)$, respectively. The vertices of $\Gamma_{G}^{\Omega}$ are non central elements in $\Omega$ but not in $A$, that is $V\left(\Gamma_{G}^{\Omega}\right)=\Omega-A$, while the number of edges are $\left|E\left(\Gamma_{G}^{\Omega}\right)\right|=\sum_{i=1}^{\left|V\left(\Gamma_{G}^{\Omega}\right)\right|}\left(\begin{array}{c}\left|v_{i}\right| \\ 2\end{array}\right)$, where $v$ is the size of orbit under group action of $G$ on $\Omega$. Two vertices $v_{1}, v_{2}$ are adjacent in $\Gamma_{G}^{\Omega}$ if $v_{1}$ and $v_{2}$ are conjugate that is, $v_{1}=g^{v_{2}}$.

The orbit of $v \in \Omega$ is $\operatorname{cl}(v)=\{v g, g \in G\}$. Hence $\Omega=\cup_{v \in \Omega} \operatorname{cl}(v)$. Since $\Gamma_{G}^{\Omega}$ represents the orbit graph whose vertices are non-central orbits, therefore $\Gamma_{G}^{\Omega}$ consists of complete components of orbits under group action on $\Omega$. As stated in the first section, the chromatic number, the clique number, the independent number, the dominating number and diameter of a graph $\Gamma$ are denoted by $\chi(\Gamma), \omega(\Gamma), \alpha(\Gamma), \gamma(\Gamma)$ and $d(\Gamma)$, respectively. The following proposition illustrates the properties of the orbit graph. 
Proposition 5. Let $G$ be a finite non-Abelian group and let $\Omega$ be a set. If $G$ acts on $\Omega$, then the properties of the orbit graph $\Gamma_{G}^{\Omega}$ are described as follows.

1. $\chi\left(\Gamma_{G}^{\Omega}\right)=\min \left\{\left|c l\left(v_{i}\right)\right|, v_{i} \in \Omega\right\}$,

2. $\omega\left(\Gamma_{G}^{\Omega}\right)=\max \left\{\left|c l\left(v_{i}\right)\right|, v_{i} \in \Omega\right\}$,

3. $\alpha\left(\Gamma_{G}^{\Omega}\right)=K(\Omega)-|A|$,

4. $\gamma\left(\Gamma_{G}^{\Omega}\right)=K(\Omega)-|A|$.

5. $d\left(\Gamma_{G}^{\Omega}\right)=\max \left\{d(v, u): \forall v, u \in V\left(\Gamma_{G}^{\Omega}\right)\right\}$.

Proof. Since the chromatic number is the minimum number of coloring vertices, thus $\chi\left(\Gamma_{G}^{\Omega}\right)=\min \left\{\left|\operatorname{cl}\left(v_{i}\right)\right|, v_{i} \in \Omega\right\}$, meanwhile the clique number is the maximum number of complete subgraphs, thus $\omega\left(\Gamma_{G}^{\Omega}\right)=\max \left\{\left|\operatorname{cl}\left(v_{i}\right)\right|, v_{i} \in \Omega\right\}$. A subset $M$ of $V\left(\Gamma_{G}^{\Omega}\right)$ is called the independent set if the induced subgraph on $M$ has no edges. Thus the independence number of the orbit graph $\alpha\left(\Gamma_{G}^{\Omega}\right)$ is the maximum size of the independent set, this can be obtained when $K(\Omega)$ $|A|$. However, a subset $T$ of vertices, $M(T)$ is the set of $V\left(\Gamma_{G}^{\Omega}\right)$ that are in $T$ or connected to a vertex in $T$. Thus $T$ is a dominating set if $M(T)=$ $V\left(\Gamma_{G}^{\Omega}\right)$, hence the the minimum size of dominating set is $K(\Omega)-|A|$. The maximum distance between any two vertices of $\Gamma_{G}^{\Omega}$ is the diameter, thus $d\left(\Gamma_{G}^{\Omega}\right)=$ $\max \left\{d(v, u): \forall v, u \in V\left(\Gamma_{G}^{\Omega}\right)\right\}$.

The following propositions are obtained.

Proposition 6. If $G$ is an Abelian group, then $\Gamma_{G}^{\Omega}$ is a null graph.

Proof. Suppose $G$ is an Abelian group, thus $g v=v g$, where $v, g \in G$. Since $\left|V\left(\Gamma_{G}^{\Omega}\right)\right|=|\Omega|-|A|$, where $A=\{v \in G \mid v g=g v, g \in G\}$, hence the graph is a null graph.

Next, we provide a theorem that is considered as a key connect between the probability that a group element fixes a set and the orbit graph.

Proposition 7. Let $G$ be a finite non-Abelian group and let $\Omega$ be the set of all subsets of commuting elements of $G$ of size two. If $G$ acts on $\Omega$ by conjugation and $P_{G}(\Omega)=1$, then $\Gamma_{G}^{\Omega}$ is a null graph.

Proof. Suppose $P_{G}(\Omega)=1$. Then by Theorem $1, K(\Omega)=|\Omega|$ thus $\operatorname{cl}(\omega)=$ $\omega$ for all $\omega \in \Omega$. Thus all elements $a$ and $b$ are in the center of $G$. Since $\left|V\left(\Gamma_{G}^{\Omega}\right)\right|=|\Omega|-|A|$ and $A=\{\omega g=g \omega, \omega \in \Omega\}$ leads to $|\Omega|=|A|$, thus the graph is null. 
The following theorem shows if two orbit graphs are isomorphic, then their sets orders are identical.

Theorem 8. Let $G$ and $H$ be finite non-Abelian groups. Let $\Omega_{G}$ and $\Omega_{H}$ be the subsets of $G$ and $H$, respectively. If $G$ acts on $\Omega_{G}$ and $H$ acts on $\Omega_{H}$ and $\Gamma_{G}^{\Omega_{G}} \cong \Gamma_{H}^{\Omega_{H}}$, then $\left|\Omega_{G}\right|=\left|\Omega_{H}\right|$.

Proof. Since $\Gamma_{G}^{\Omega_{G}} \cong \Gamma_{H}^{\Omega_{H}}$, it follows that $\left|V\left(\Gamma_{G}^{\Omega_{G}}\right)\right|=\left|V\left(\Gamma_{H}^{\Omega_{H}}\right)\right|$ and $\left|E\left(\Gamma_{G}^{\Omega_{G}}\right)\right|=$ $\left|E\left(\Gamma_{H}^{\Omega_{H}}\right)\right|$. Using Definition 4,

$$
\left|\Omega_{G}\right|-\left|A_{G}\right|=\left|\Omega_{H}\right|-\left|A_{H}\right| \text { and } \sum_{i=1}^{K\left(\Omega_{G}\right)-\left|A_{G}\right|}\left(\begin{array}{c}
\mid x^{\Omega_{G_{i}} \mid} \\
2
\end{array}\right)=\sum_{i=1}^{K\left(\Omega_{H}\right)-\left|A_{H}\right|}\left(\begin{array}{c}
\left|x^{\Omega_{H_{i}}}\right| \\
2
\end{array}\right) .
$$

Therefore $\left|A_{G}\right|=\left|A_{H}\right|$ and thus $\left|\Omega_{G}\right|=\left|\Omega_{H}\right|$. The proof then follows.

The following proposition shows the disconnectivity of orbit graph.

Proposition 9. The orbit graph $\Gamma_{G}^{\Omega}$ is not connected.

Proof. Assume that $\Gamma_{G}^{\Omega}$ is a connected graph. Thus, there exists $v_{1}, v_{2} \in$ $V\left(\Gamma_{G}^{\Omega}\right)$ such that $v_{1}$ is adjacent to $v_{2}$. There exists an element $g \in G$, such that $g v_{1}=v_{2}$. Since $\Omega$ is a disjoint union of distinct orbits, there is an element $k \in \Omega$ such that $k$ is not adjacent to $v_{1}$ or $v_{2}$. Therefore $\Gamma_{G}^{\Omega}$ is connected, a contradiction.

Next, we prove the orbit graph is not a bipartite graph.

Theorem 10. Let $G$ be a finite non-Abelian group. Let $\Omega$ be the set of all subsets of commuting elements of $G$ of size two. If $G$ acts on $\Omega$, then the orbit graph $\Gamma_{G}^{\Omega}$ is not a complete bipartite graph.

Proof. Suppose $\Gamma_{G}^{\Omega}$ is a complete bipartite orbit graph. Thus the number of vertices $\left|V\left(\Gamma_{G}^{\Omega}\right)\right|=\left|V_{1} \cup V_{2}\right|$, where $V_{1}$ and $V_{2}$ are two sets of vertices. Therefore, $|\Omega|-|A|=\left|V_{1}\right|+\left|V_{2}\right|$. Assume that $\left|V_{1}\right| \leq\left|V_{2}\right|$, thus $\left|V_{1}\right| \leq \frac{1}{2}\left(\left|V_{1}\right|+\left|V_{2}\right|\right)$. Take $\omega \in V_{2}$, thus from the completeness of the bipartite graph, the degree of $V$ is $\operatorname{deg}(\omega)=\left|v_{1}\right| \leq \frac{1}{2}(|\Omega|-|A|)$. Since $G$ acts on $\Omega$ thus $|G|-\left|C_{\Omega}(\omega)\right|=\operatorname{deg}(\omega) \leq$ $\frac{1}{2}(|G|-|A|)$. Therefore, $\frac{|G|}{2} \leq\left|C_{\Omega}(\omega)\right|-\frac{|A|}{2}$. Since $|A|$ divides $\left|C_{\Omega}(\omega)\right|$, there exists $k$ such that $\frac{|G|}{2} \leq\left(k-\frac{1}{2}\right)|A|$. If $A=C_{\Omega}(\omega)$, then $\omega \in C_{\Omega}(\omega)=A$, a contradiction. Hence, $|A| \leq \frac{\left|C_{\Omega}(\omega)\right|}{2}$, and $\frac{|G|}{\left|C_{\Omega}(\omega)\right|}=\frac{|G|}{k|A|} \leq \frac{1}{k}(2 k-1)=2-\frac{1}{k}<2$, it follows that $\frac{|G|}{\left|C_{\Omega}(\omega)\right|}=1$. This implies that $|G|=C_{\Omega}(\omega)$, a contradiction. Thus, $\Gamma_{G}^{\Omega}$ is not a complete bipartite graph. 
Next, we determine the orbit graph of dihedral groups and quaternion groups, where $\Omega$ here is the set in Theorem 1. Starting with dihedral groups.

Theorem 11. Let $G$ be a dihedral group of order $2 n$. If $G$ acts on $\Omega$ by conjugation, then

$$
\Gamma_{G}^{\Omega}=\left\{\begin{array}{l}
\cup_{i=1}^{5} K_{\frac{n}{2} i}, n \text { is even and } \frac{n}{2} \text { is odd } \\
\left(\cup_{i=1}^{4} K_{\frac{n}{2} i}\right) \cup\left(\cup_{i=1}^{2} K_{\frac{n}{4}} i\right), n \text { and } \frac{n}{2} \text { are even, } \\
K_{n}, n \text { is odd. }
\end{array}\right.
$$

Proof. Based on Theorem 2, the number of elements in $\Omega$ is $\frac{5 n}{2}+1$ and using Definition 4 , the number of vertices of $\Gamma_{G}^{\Omega}$ is $\frac{5 n}{2}$. Two vertices $\omega_{1}$ and $\omega_{2}$ are adjacent if $\omega_{1}=\omega_{2}^{g}$. First, when $n$ is even and $\frac{n}{2}$ is odd. there are five complete components of $K_{\frac{n}{2}}$, since there are six orbits five of them are of size $\frac{n}{2}$. Second, when $n$ and $\frac{n}{2}$ are even. The orbit graph $\Gamma_{G}^{\Omega}$ consists of four complete components of $K_{\frac{n}{2}}$ and two complete components of $K_{\frac{n}{4}}$, represented to four orbits of size $\frac{n}{2}$ and two orbits are of size $\frac{n}{4}$. However, when $n$ is odd there is only orbit of size $n$, hence $\Gamma_{G}^{\Omega}$ consists of one complete component of $K_{n}$. The proof then follows.

The orbit graph of quaternion groups.

Theorem 12. Let $G$ be a quaternion group of order $2^{n}$. If $G$ acts on $\Omega$ by conjugation, then $\Gamma_{G}^{\Omega}$ is a null graph.

Proof. According to Theorem $3, P_{G}(\Omega)$ is equal to one since there is only one orbit of size one. By Definition 4, the number of vertices is zero. Using Proposition $7, \Gamma_{G}^{\Omega}$ is a null graph.

\section{Conclusion}

In this paper, we introduced a new graph called orbit graph whose vertices are non-central orbits under group action on a set. Besides, some properties of the orbit graph were determined. The orbit graph is found for dihedral groups and quaternion groups. 


\section{Acknowledgment}

The first author would like to acknowledge Ministry of Higher Education in Libya.

\section{References}

[1] J. Bondy, G. Murty, Graph Theory with Application, North Holand, Boston New York, 5th (1982).

[2] C. Godsil, G. Royle, Algebraic Graph Theory, Springer, Boston New York, 5th (2001).

[3] G.A. Miller, Relative Number of Non-invariant Operators in a Group, Proc. Nat. Acad. Sci., USA, 30(20), (1994), 25-28.

[4] S.M.S. Omer, N.H. Sarmin, A. Erfanian and K. Moradipour, The Probability That an Element of a Group Fixes a Set and the Group Act on Set by Conjugation, International Journal of Applied Mathematics and Statistics., 32(2) (2013), 111-117.

[5] S.M.S. Omer., N.H. Sarmin and A. Erfanian, The Probability that an Element of a Symmetric Group Fixes a Set and Its Application in Graph Theory, World Applied Sciences Journal., 27(12) (2013), 1637-1642.

[6] S.M.S. Omer, N.H. Sarmin and A. Erfanian, The Probability That an Element of a Group Fixes a Set and Its Graph Related to Conjugacy Classes, Journal of Basic and Applied Scientific Research., 3(10) (2013), 369-380.

[7] E.A. Bertram, M. Herzog and A. Mann, On a Graph Related to Conjugacy Classes of Groups, Bull. London Math. Soc., 22(6) (1990), 569-575.

[8] M. Bianchi, M. Herzog, E. Pacifici and G. Saffirio, On the Regularity of a Graph Related to Conjugacy Classes of Groups, European Journal of Combinatorics., 33(7) (2012), 1402-1407.

[9] K. Moradipour, N.H. Sarmin and A. Erfanian, On Graph Associated to Conjugacy Classes of Some Metacyclic 2-Groups, Journal of Basic and Applied Scientific Research, 3(1) (2013), 898-902. 
[10] A. Erfanian, B. Tolue, Conjugate Graphs of finite Groups, Discrete Mathematics, Algorithms and Applications, 4(2) (2012), 35-43. 
